\title{
EPIGENETICS IN PSYCHIATRY
}

Nupur Mahatme ${ }^{1}$, Manoj Shettar ${ }^{2}$, Anil Kakunje ${ }^{1}$, Ganesh Kini ${ }^{1}$

1- Yenepoya Medical College Hospital, Mangalore, 2-SDM Medical College Dharwad

\begin{abstract}
Epigenetics is defined as the changes in gene expression that lead to predictable, stable and possibly heritable alterations in cell function that are not due to changes in DNA sequence. It is of major relevance in psychiatry since environmental factors like stress or substance abuse, significant contribute in causing psychiatric pathologies like depression, anxiety, depression, etc.

Epigenetics research in psychiatry would give an opportunity to extend the outlook and approach towards psychiatric pathologies. Hence timely interventions would be sought for. This would also allow gaining access to 'brain tissue' from live patients with mental illness and predicting occurrence of or vulnerability to psychiatric illnesses by evaluation of biomarkers, thus preventing, reversing or atleast curbing the progress at an initial stage.

There are evidences of work done in the field of epigenetics both in pre-clinical as well as clinical settings. It is remarkable to see how the DNA methylation at various sites can result in alteration of the mental health of an individual and also how this can affect as early as during the first week of life. This is an exciting frontier in psychiatry, providing long sought biomarkers for predicting complex and devastating human behaviours as well as mental illnesses.
\end{abstract}

Keywords: Epigenetics, psychiatry, gene-environmental interaction, vulnerability, psychiatric pathologies.

\section{Corresponding author:}

Nupur Mahatme

Department of Psychiatry

Yenepoya Medical College Hospital, Mangalore

Email: nupur.mahatme@gmail.com 


\section{Introduction}

The term "epigenetics" was coined by fusing the two terms "genetics" and "epigenesis" by Conrad Waddington in 1942. This term was used to describe the developmental program in a multi-cellular organism wherein the zygote gives rise to different cell types. Waddington defined it as "the cooperation between genetic traits and environmental conditions that together established a stable cellular phenotype." Others also defined it as a stably heritable phenotype resulting from changes in a chromosome without alterations in the DNA sequence. ${ }^{1}$

The current definition used is "Changes in gene expression that lead to predictable, stable and possibly heritable alterations in cell function that are not due to changes in DNA sequence." In psychiatry, this definition is particularly relevant since environmental factors, such as stress or substance abuse, alter the expression of genes in post-mitotic neurons that are not heritable, but significantly contribute in causing psychiatric pathologies such as depression, anxiety and addiction. ${ }^{1}$

Different cell types can be generated by using the same genetic blueprint by mechanisms that are involved in epigenetics. The genetic code as well as the DNA sequence remains unchanged, however two different epigenetic programs read it differently, depending on what letters within the underlying code are more visible. ${ }^{1}$

\section{Epigenetics in psychiatry}

Epigenetics research is one of the most exciting and rapidly growing frontiers in psychiatric research. This is due to its potential to advance the field in six specific areas: ${ }^{1}$

1) How environmental factors shape vulnerability to psychiatric conditions is explained by molecular mechanisms. For example, in cases of substance use, post-traumatic stress disorder (PTSD), contribution of environmental factors is quite obvious. In other examples like schizophrenia and bipolar disorder, a strong environmental influence can be there since concordance between monozygotic twins is slightly less than $50 \%$. It is also important to recognize that environmental manipulations can induce epigenetic changes that can alleviate psychiatric symptoms. In fact, many of the known interventions in psychiatry such as antidepressant medications, ECT, diet, exercise have shown to cause epigenetic changes that modify vulnerability to stress, anhedonia and drug seeking behaviours.

2) It can also explain how abnormal brain development can contribute to the vulnerability to many of the psychiatric conditions. We will further see how epigenetics plays a crucial role in modifying brain development and vulnerability to various psychopathologies in children exposed to abuse or neglect; thus, targeting for timely interventions.

3)Somatic cells can be harvested by researchers through this technique (eg skin cells) from individuals diagnosed with specific psychiatric illnesses (eg schizophrenia) and further their epigenetic code can be reprogrammed in a way that would transform them into neurons or glial cells, which can then be grown and studied in a culture. This allows gaining access to 'brain tissue' from live patients with mental illness.

4) Heritability is calculated as the relative rates of concordance between monozygotic and dizygotic twins, and monozygotic twins share many more epigenetic similarities comparatively. Also the ability of some epigenetic markers to be inherited may explain how exposure to a specific stressor in the parental generation may modify the risk for psychopathology in the offspring.

5) Such gene-by-environment interactions have been demonstrated between alleles of the serotonin transporter and the ability of childhood maltreatment to modify risk of adult depression, elucidating the mechanism by which gene and environmental factors interact to modify vulnerability to mental illness, thus introducing more specific biomarkers and early interventions.

6) Provides reliable biomarkers are provided to diagnose psychiatric vulnerabilitiessuch as DNA methylation. Epigenetic changes can be a potentially valuable biomarker for diagnosing and assessing treatment response. Biomarkers are desperately needed in psychiatry where no objective disease markers are currently available to diagnose or assess treatment response. ${ }^{1}$

\section{Epigenetic Modifications}

The chemical structure of DNA as well as the amino acids of histones can undergo modifications such as methylation, phosphorylation, acetylation, etc. These 
processes are collectively called as epigenetic modifications, which are reversible and are carried out by large multi-subunit protein complexes that coordinate their activities that can either condense or open up the chromatin in response to the environmental cues. ${ }^{1}$

\section{Molecular mechanisms of epigenetics:}

It includes DNA methylation, histone modification and RNA-associated silencing. ${ }^{1}$

DNA methylation depending on the position of the methylation change relative to the position of the transcription start site, inhibits gene transcription.DNA gets methylated at sites where there is cytosine by the enzymatic addition of a methyl group, using $\mathrm{S}$ adenosyl methionine (SAM) as the methyl group source, to the carbon-5 position of cytosine. ${ }^{2}$ Epigenetic alterations occur through DNA methylation of cytosine-guanine base $(\mathrm{CpG})$ dinucleotides using DNA methyltransferases (DNMT1, 3a, 3b). ${ }^{3}$

DNA methylation has functions like silencing of transposable elements, defense against viral sequences and transcriptional repression of certain genes. ${ }^{4}$

Studies on mice have shown that epigenetic modification through cytosine methylation and chromatin remodeling may get inherited to the next generations. ${ }^{5}$

The most abundant proteins in chromatin are histones. Histones can be modified through acetylation, methylation, and phosphorylation of the histone tail domains. Histone modifications results in dynamic transitions between gene activation and silencing. ${ }^{6}$

Enzymes involved in histone modification are histone acetyl transferases (HATs) and histonedeacetylases (HDACs). These modifications show effect on gene expression by altering the structural configuration of the genome. ${ }^{3}$

Small RNAs can silence genes at transcriptional and post-transcriptional levels. Small RNAs have 21 to 28 nucleotides. Small RNAs include micro RNAs (miRNAs) and small interfering RNA (siRNAs). RNA-mediated gene silencing is required for maintaining chromosomal structure, genome defense and gene regulation. ${ }^{7}$

\section{Environmental factors:}

The environmental factors have an impact on epigenetic mechanisms in gene expression by many ways.Dietary levels of methionine, during prenatal and early postnatal life, influences susceptibility to chronic adult diseases because dietary methionine influences DNA methylation. ${ }^{8}$

Environmental factors can influence the epigenome. Pup licking and grooming (LG) and arched-back nursing $(\mathrm{ABN})$ by rat mothers alters the offspring epigenome at a glucocorticoid receptor (GR) gene promoter in the hippocampus. Offspring of mothers that showed high levels of LG and ABN had differences in DNA methylation, as compared to offspring of 'low-LG-ABN' mothers. ${ }^{9}$

\section{Psychosocial factors:}

Psychosocial factors like maternal behavior and stress reactivity in the offspring, transmission of such individual differences in maternal behavior from one generation of females to the next, environmental manipulation occurring during early development which alters maternal behavior can then affect the pattern of transmission in subsequent generations. ${ }^{10}$

\section{Epigenetic inheritance:}

It is by three methods: Mitotic inheritance, meiotic inheritance and true transgenerational inheritance. ${ }^{11}$

Inheritance of the epigenome is a powerful tool by which adaption of one generation of organisms to a changed environment can be carried to many subsequent generations. This can result in either adaptive or negative effects in later generations. The classical twin study first proposed by Galton shows that there is a higher concordance rate between MZ twins compared with dizygotic (DZ) twins provides evidence for genes influencing phenotypes. MZ twins discordant for a trait are given as example for environmental factors influence in complex disease aetiology. ${ }^{12}$

\section{Pre-clinical work in epigenetics}

Epigenetic transmission of Stress reactivity and maternal behaviour -

The importance of early life experiences for the development of the organism has long been appreciated from both psychodynamic and biologic perspectives. Cases of early life abuse and neglect 
often fit into a trans-generational pattern in which childhood maltreatment is associated with maladaptive parenting styles later in life. Exposure to early life stress is linked with enhanced endocrine and autonomic responses to stress that persist into adulthood. This increased stress reactivity is closely related to increased risk for developing several psychopathologies including affective disorders, PTSD and substance use. ${ }^{1}$

Biological mechanisms were investigated by studying the parental styles in rats. It was observed that parental neglect increases stress reactivity in a manner that persists even into adulthood and further can also be transmitted across generations. Some of the mothers licked and groomed their pups almost three times more frequently compared to others during the first postpartum week, they have found to have profound behavioral phenotypes. For example off springs raised by high lick and groom (LG) mothers performed better in several memory tests, and were less fearful and showed decreased corticosteroid secretion in response to stress as compared to neglected offspring raised by low LG mothers. These behavioural changes are due to the frequency of LG during the first week of life and not due to genetic differences between them. Cross fostering studies have shown that offspring born to low LG mothers but raised by high LG mothers were behaviorally indistinguishable from pups born and raised by high LG mothers. ${ }^{1,2}$

Rat pups were provided with different amount of paintbrush strokes which could mimic maternal behavior during the first week of life induced similar behavioural changes which persisted even in adulthood, indicating that exposure to different levels of tactile stimulation during the first week of life in some way programs adult behavior.

The frequency of LG during the first week of life causes stable changes in DNA methylation in promoter elements that regulate the expression of genes. For example, the promoter of glucocorticoid receptor (GR) is heavily methylated at birth, thus leading to low GR levels in the hippocampus of newborn pups. Demethylation of this GR promoter allows transcription activator NGF inducible protein A. ${ }^{1}$

Cortisol is produced in response to stress. The more stressed we are the more cortisol we produce. The average level of cortisol production tends to be raised in adults who had traumatic childhoods, even if they are healthy at the time of measurement. This shows that higher background stress levels are found in adults who were abused or neglected as their contemporaries. $^{2}$

The development of mental illness is little like the development of cancer that a lot has to go wrong at the molecular level before a person becomes clinically ill. This increases their vulnerability to the disease. How does this over-expression of cortisol happen? There is a whole signaling cascade involved in here. During an abusive childhood, this signaling cascade is very active. In many abuse survivors, this system keeps signaling as if the person is still trapped in the abusive situation. It's as if the thermostat on a central heating system has malfunctioned, and the boilers and radiators continue to pump out heat in April, based on the weather from previous November. ${ }^{2}$

The process starts in a region of the brain called the hippocampus, where in because of changes in its activities in response to stress, the hypothalamus produces and releases two hormones, called corticotrophin releasing hormone and arginine vasopressin. These hormones stimulate the pituitary gland, which responds by releasing adrenocorticotropin hormone which gets into the bloodstream and when the cells of the adrenal gland take up this hormone, they release cortisol..$^{2-4}$ Most important to epigenetic stress is that glucocorticoids are a prominent regulator of Hypothalamo-PituitaryAdrenal (HPA) axis activation via negative feedback. $^{5}$

Also it has been observed that increased LG behavior results in decreased DNA methylation at estrogen receptor $-\alpha(E R \alpha)$ in medial pre-optic area (MPOA) in hypothalamus, which in turn recruits transcription factor Stat5, thus increasing ER $\alpha$ in medial pre-optic area in increased female LG offspring, subsequently improving maternal behaviour. These modifications can however be reversed by IV infusion of Histone deacetylase (HDAC) inhibitor which reduces the DNA methylation at GR promoter, thus increasing GR levels in hippocampus and decreasing stress reactivity in adult low LG offsprings. Similarly IV infusion of Methionine, increases DNA methylation at GR promoter, decreasing GR levels in hippocampus and in turn increasing stress reactivity in adult high LG offsprings. ${ }^{1}$ 
Epigenetic Inheritance of Olfactory Fear Memory -

A critical survival function in both animals and humans is the ability to recognise, remember and avoid dangerous environmental signals. While humans rely strongly on visual cues, rodents scout their environment with their highly sensitive sense of smell. ${ }^{1}$

In mice, the repeated pairing of a specific odour referred as the "offending odour", with a noxious stimulus such as foot shock, results in a fear memory that is manifested as an exaggerated startle when the rodent encounters the offending odour alone without the shock. Olfactory mediated memory is therefore considered as an adaptive form of sensitisation to environmental threats that help the mouse to predict and avoid danger.To test the inheritance of sensitivity to danger associated olfactory stimuli, mice were fear conditioned to a specific conditioned odor. This procedure was done in mice in which the neurons expressing the olfactory receptor coding for that specific offending odor were labeled with a LacZ reporter. The advantage of this allows the researcher to assess whether the sensitivity to the offending odor is accompanied by an increase in the expression of its receptor in the olfactory epithelium. ${ }^{1}$

Neuroanatomical examination of $\mathrm{F} 1$ and $\mathrm{F} 2$ mice showed that expression of the olfactory receptor responsive to the conditioned odor was selectively upregulated in the olfactory epithelium. To detect if this transgenerational transmission was due to epigenetic modifications, parental sperm was collected and IVF was done. DNA isolated from sperm of F0 and F1 showed hypomethylation in the vicinity of olfactory receptor responsible for that specific odor and not around others.

Epigenetic Mechanisms of Depressive Behaviour and Anti-depressant Action -

Rodent models of depression and PTSD typically employ some form of chronic, unpredictable or severe stress that leads to despair, avoidance or anhedonic-like behaviours. These behavioural consequences could be reversed with chronic treatment with antidepressants, thus providing reasonable models of human depression and PTSD. ${ }^{1}$

One paradigm involves chronic defeat in which a mouse is briefly exposed to an aggressive intruder over the course of 10 days and the defeated mice shows decrease in sucrose consumption, suggesting an anhedonic-like state, increased helplessness in the forced swim and tail suspension tests. Behavioral consequences of social defeat and the rescue of depressive phenotype by antidepressant medications are due to epigenetic changes in the expression of the genes implicated in neuroplasticity. One of the best eg of epigenetic regulation of neuroplasticity genes is the opposing effects of stress and antidepressants on the expression of brain derived neurotropic factor (BDNF) in limbic brain areas. Antidepressants reverse the effects produced in defeated mice due toraised $\mathrm{H} 3 \mathrm{~K} 27$ methylation at BDNF promoter. Repressive chromatin state inhibits BDNF expression and thus downregulates expression of BDNF in hippocampus. ${ }^{1}$

In a study done by Natalia Onishchenko et al; it was seen that perinatal exposure of mice to Methyl Mercury can lead to long-lasting depression-like behavioural changes which was associated with repressive epigenetic changes in the regulation of the BDNF gene expression. When same mice during adulthood were treated with Fluoxetine chronically; the depressive symptoms improved by reversing the effect of methyl mercury on behaviour and BDNF gene expression. ${ }^{13}$

The acetylating agent L-acetylcarnitine (LAC), an antidepressant acts through epigenetic regulation of type 2 metabotropic glutamate (mGlu2) receptors. In studies it has caused rapid and long-lasting antidepressant effect in Flinders Sensitive Line rats and in mice exposed to chronic unpredictable stress, which, respectively, represent genetic and environmentally induced depression. LAC increased the levels of acetylated H3K27 bound to the Grm2 promoter and also increased acetylation of NF-KBp65 subunit, thereby intensifying the transcription of Grm2 gene encoding for the mGlu2 receptor in hippocampus and prefrontal cortex. LAC reduced the immobility time in the forced swim test and increased sucrose preference in 3 days of treatment, the antidepressant effect was seen 2 weeks after drug withdrawal. $^{14}$

Epigenetic Mechanisms of Cocaine Addiction -

Positive experiences can also be epigenetically coded. Maladaptive compulsive drug seeking behaviour can occur when the natural rewarding system has been hijacked by highly rewarding exogenous substances. ${ }^{1}$ 
In rodents, the transition to addictive behaviour is mediated by epigenetic changes in the expression of genes that modify synaptic plasticity in neurons located in the reward system. Initial effects of cocaine cause transient expression of immediate early genes such as c-fos, whereas chronic use of cocaine increases the expression of late onset genes such as FosB/AFosB, CDK5, BDNF.Histone acetylation plays a critical role in coordinating the transition from inducing immediate early genes to the stable expression of late onset genes. Manipulations that inhibit histone acetylation such as viral knockdown of HAT or the over-expression of different HDACs in the nucleus accumbens block the ability of chronic cocaine to induce late onset genes and reduce compulsive drug seeking behaviors. ${ }^{1}$

Epigenetic mechanisms in Memory -

In mammals, both DNA methylation and histone modifications play a role in learning and memory. DNA methyltransferase proteins DNMT3A and DNMT3B increase in expression in adult rat hippocampus in a particular learning and memory model. Conversely, treating these rats with a DNA methyltransferase inhibitor such as 5-azacytidine blocks memory formation and affects both the hippocampus and the cortex. Mice whose neurons over-express HDAC2 have poor long term memory. Increased acetylation levels in the brain seem to be consistently associated with improved memory. ${ }^{2}$

\section{Epigenetics in clinical setting}

\section{Fragile X Syndrome}

It is anX-linked monogenetic neuro-developmental psychiatric disorder, characterised by moderate intellectual disability, social deficits, a high incidence of attention deficit hyperactivity disorder (ADHD) and multiple physical abnormalities. It is caused by loss of function of the fragile $\mathrm{X}$ mental retardation gene 1 (FMR1). The FMR1 gene codes for an RNA binding protein that plays an important role in dendritic spine formation, explaining its impact on cognitive function and behaviour.DNA methylation is necessary to induce the clinical syndrome in individuals with full mutation. Hence Therapies preventing DNA methylation could potentially rescue the neurodevelopmental sequelae of individuals with fully mutated allele. ${ }^{1}$

Preliminary experiments using a lymphoblastoid cell line have been used to demethylate the fully mutated promoter and induce protein expression using a combination of DNMT and HDAC inhibitors. ${ }^{1}$

\section{Post-traumatic stress disorder}

Effects of stress on complex behaviours are mediated by genetic vulnerability. Childhood trauma preferentially interacts with a specific genetic predisposition to increase expression of the FK506binding protein 5 (FKBP5) and vulnerability to PTSD and depression. ${ }^{1}$

FKBP5 is a stress related gene that exerts its effects via interaction with the GR. Under basal conditions, GR is maintained in the cytoplasm by the heat shock 90 and FKBP5 proteins, which bind GR and mask its nuclear localization signal. High levels of cortisol induced by stress dissociate Hsp90 and FKBP5 from GR, allowing it to enter the nucleus and induce 2 transcriptional programs. The first, is essential for terminating further release of cortisol from the adrenal gland, and second involves induction of FKBP5, a negative feedback loop that prevents further entry of GR into the nucleus. Retaining Gr in the cytoplasm is thought to reset the stress response so it can be reactivated in response to additional threats. ${ }^{1}$

\section{Suicide Biomarker}

Recent findings show that there are certain peripheral epigenetic markers that can predict suicide with more than $80 \%$ accuracy hence these tragedies can be prevented. The DNA methylation patterns from the prefrontal cortex of depressed patients who committed suicide were studied as compared with depressed patients who died of other causes. DNA methylation is amenable to study in post mortem tissue because of its relative stability and its ability to predict gene expression. Only one gene coding for the spindle and kinetochore associated complex subunit 2 (SKA2) was identified to be differentially methylated in neurons of suicide committers.SKA2 is a scaffolding protein that chaperones the GR into the nucleus, thus terminating HPA activation in response to stress. It is a reliable retrospective biomarker of suicide. ${ }^{1}$

In addition, levels of SKA2 methylation in immune cells were highly correlated with morning cortisol levels suggesting a possible functional link between SKA2 methylation and stress levels. In some cases high levels of methylation at SKA2 were detected months before the onset of suicidal ideation, thus 
raising the possibility that this biomarker was present even before they developed suicidal thoughts. ${ }^{1}$

The inclusion of additional variables such as cortisol levels and anxiety further improved the predictive value of the model. This model was able to predict suicidal behavior with $71 \%$ accuracy. Accuracy was increased to $82 \%$ when levels of salivary cortisol were added. Predictive value further improved to $97 \%$ on refining the stringency of categorizing suicidal ideations. ${ }^{1}$

In one study, patients who had suffered from childhood maltreatment, especially physical abuse, had high rates of DNA methylation of specific $\mathrm{CpG}$ sites in the glucocorticoid response element region of the 5-HT3AR, which may be associated with the frequency of suicide attempts and hospitalizations. ${ }^{1}$

\section{Borderline Personality Disorder}

The DSM-5 defines borderline personality disorder as "a pattern of instability in interpersonal relationships, self-image, and affects, and marked impulsivity". 6 There are multiple studies that indicate that childhood maltreatment, specifically sexual abuse, is both commonly reported in and strongly associated with borderline personality disorder, with symptoms of the personality disorder, such as derealization or dysphoria, predictive of childhood abuse. ${ }^{7-9}$ Individuals with a history of childhood physical abuse, sexual abuse or neglect were four times more likely to have a personality disorder in early adulthood compared with individuals who did not have such a history. ${ }^{10,13}$

Glucocorticoid receptor gene NR3C1 is a target of early life stress because increased methylation of its promoter region in exon $1 \mathrm{~F}$ has been documented in both, patients with childhood trauma and patients with borderline personality disorder. ${ }^{14-16}$

\section{Atypical Antipsychotic Regulation}

Histone deacetylases (HDACs) compact chromatin structure and represses gene transcription. ${ }^{17}$ In schizophrenia, preclinical ${ }^{18-20}$ and clinical 21-23 studies suggest that drugs such as valproate, one of whose functions is to act as a nonspecific histone deacetylase (HDAC) inhibitor ${ }^{24,25}$ are efficacious when given chronically in combination with atypical antipsychotic drugs. Chronic atypical antipsychotics down-regulates the transcription of metabotropic glutamate 2 receptor (mGlu2), an effect that is associated with decreased histone acetylation at its promoter in mouse and human frontal cortex, ${ }^{26}$ a brain region that is important in cognition and perception, and has been implicated more recently in schizophrenia and antipsychotic responses. ${ }^{27,28}$

This epigenetic change occurred in concert with a serotonin 5-HT2A receptor-dependent up-regulation and increased binding of HDAC2 to the mGlu2 promoter.Virally mediated over-expression of HDAC2 in frontal cortex decreased mGlu2 transcription, thereby increasing psychosis like behaviour. $^{17}$

HDAC inhibitors (sodium valproate) prevent the repressive histone modifications induced at the mGlu2 promoter by atypical antipsychotics and augment their therapeutic effects. ${ }^{24,25}$

\section{Conclusion}

Epigenetics is such an exciting frontier in psychiatry, providing long sought biomarkers for predicting complex and devastating human behaviours as well as mental illnesses.

\section{References:}

1. Jeniffer B Dwyer, Arie Kaffman. Epigenetics in Psychiatry: The promise for New Biomarkers and Treatments. In: Benjamin James Sadock, Virginia Alcott Sadock, Pedro Ruiz (Eds). Kaplan \& Sadock's Comprehensive Textbook of Psychiatry. Tenth Edition. Philadelphia: Wolter Kluwer; 2017.p 386-400.

2. Carey N. The epigenetics revolution: How modern biology is rewriting our understanding of genetics, disease, and inheritance. Columbia University Press; 2012.

3. Vale W, Spiess J, Rivier C, Rivier J. Characterization of a 41-Residue Ovine Hypothalamic Peptide that Stimulates Secretion of Corticotropin and $\beta$-endorphin. Science. 1981;213(4514):1394-7.

4. Rivier C, Vale W. Modulation of stressinduced ACTH release by corticotropinreleasing factor, catecholamines and vasopressin. Nature. 198;305(5932):325-7.

5. Keller-Wood ME, Dallman MF. Corticosteroid inhibition of ACTH secretion. Endocr Rev. 1984;5(1):1-24. 
6. Regier DA, Narrow WE, Clarke DE, Kraemer HC, Kuramoto SJ, Kuhl EA, et al. DSM-5 Field Trials in the United States and Canada, Part II: Test-Retest Reliability of Selected Categorical Diagnoses. Am JPsychiatry 2013;170(1):59-70.

7. Ogata SN, Silk KR, Goodrich S, Lohr NE, Westen D, Hill EM. Childhood sexual and physical abuse in adult patients with borderline personality disorder. Am J Psychiatry. 1990;147(8):1008-13.

8. Herman JL, Perry C, Van der Kolk BA. Childhood trauma in borderline personality disorder. Am J Psychiatry. 1989;146(4):490.

9. Zanarini MC, Gunderson JG, Marino MF, Schwartz EO, Frankenburg FR. Childhood experiences of borderline patients. Comprehensive Psychiatry. 1989;30(1):18-25.

10. Charoensook J. Is Epigenetic Stress the Link Between Childhood Maltreatment and Borderline Personality Disorder? American Journal of Psychiatry Residents' Journal. 2017;12(6):2-4.

11. Trerotola M, Relli V, Simeone P, Alberti S. Epigenetic inheritance and the missing heritability. Human genomics. 2015 Dec;9(1):17.

12. Galton, F. (1883) "History of Twins" in Human Faculty and its Development, McMillan and Co.

13. Onishchenko N, Karpova N, Sabri F, Castrén E, Ceccatelli S. Long-lasting depression-like behavior and epigenetic changes of BDNF gene expression induced by perinatal exposure to methylmercury. Journal of neurochemistry. 2008 Aug;106(3):1378-87.

14. Perroud N, Paoloni-Giacobino A, Prada P, Olié E, Salzmann A, Nicastro R, et al. Increased methylation of glucocorticoid receptor gene $(N R 3 C l)$ in adults with a history of childhood maltreatment: a link with the severity and type of trauma. Translational Psychiatry. 2011;1(12):e59.

15. Martín-Blanco A, Ferrer M, Soler J, Salazar J, Vega D, Andión O, et al. Association between methylation of the glucocorticoid receptor gene, childhood maltreatment, and clinical severity in borderline personality disorder. Journal of Psychiatric Research. 2014;57:3440.
16. Radtke KM, Schauer M, Gunter HM, RufLeuschner M, Sill J, Meyer A, et al. Epigenetic modifications of the glucocorticoid receptor gene are associated with the vulnerability to psychopathology in childhood maltreatment. Translational Psychiatry. 2015;5(5):e571.

17. Kurita M, Holloway T, García-Bea A, Kozlenkov A, Friedman AK, Moreno JL, et al. HDAC2 regulates atypical antipsychotic responses through the modulation of mGlu2 promoter activity. Nature Neuroscience. 2012;15(9):1245-54.

18. Dong E, Guidotti A, Grayson DR, Costa E. Histone hyperacetylation induces demethylation of reelin and 67-kDa glutamic acid decarboxylase promoters. PNAS. 2007;104(11):4676-81.

19. Dong E, Nelson M, Grayson DR, Costa E, Guidotti A. Clozapine and sulpiride but not haloperidol or olanzapine activate brain DNA demethylation. PNAS. 2008;105(36):13614-9.

20. Abel T, Zukin RS. Epigenetic targets of HDAC inhibition in neurodegenerative and psychiatric disorders. Curr Opin Pharmacol. 2008;8(1):57-64.

21. Citrome L, Casey DE, Daniel DG, Wozniak P, Kochan LD, Tracy KA. Adjunctive Divalproex and Hostility Among Patients With Schizophrenia Receiving Olanzapine or Risperidone. Psychiatr Serv. 2004;55(3):2904.

22. Kelly DL, Conley RR, Feldman S, Yu Y, McMahon RP, Richardson CM. Adjunct Divalproex or Lithium to Clozapine in Treatment-Resistant Schizophrenia. Psychiatr Q. 2006;77(1):81-95.

23. Suzuki T, Uchida H, Takeuchi H, Nakajima S, Nomura K, Tanabe A, et al. Augmentation of atypical antipsychotics with valproic acid. An open-label study for most difficult patients with schizophrenia. Human Psychopharmacology: Clinical and Experimental. 2009;24(8):628-38.

24. Löscher W. Valproate: a reappraisal of its pharmacodynamic properties and mechanisms of action. Progress in Neurobiology. 1999;58(1):31-59.

25. Gurvich N, Tsygankova OM, Meinkoth JL, Klein PS. Histone Deacetylase Is a Target of Valproic Acid-Mediated Cellular 
Differentiation. Cancer Res. 2004;64(3):107986.

26. González-Maeso J, Ang RL, Yuen T, Chan P, Weisstaub NV, López-Giménez JF, et al. Identification of a serotonin/glutamate receptor complex implicated in psychosis. Nature. 2008;452(7183):93-7.

27. González-Maeso J, Sealfon SC. Psychedelics and schizophrenia. Trends in Neurosciences. 2009; 32(4):225-32.

28. González-Maeso J, Weisstaub NV, Zhou M, Chan P, Ivic L, Ang R, et al. Hallucinogens Recruit Specific Cortical 5-HT2A ReceptorMediated Signaling Pathways to Affect Behavior. Neuron. 2007;53(3):439-52. 